\title{
Some inequalities for special functions
}

\section{Ayman Shehata*}

\section{${ }^{*}$ Correspondence:} drshehata2006@yahoo.com Department of Mathematics, Faculty of Science, Assiut University, Assiut, 71516, Egypt

Department of Mathematics, College of Science and Arts in Unaizah, Qassim University, Qassim, 10363, Kingdom of Saudi Arabia

\begin{abstract}
This paper is motivated by an open problem of several inequalities for confluent hypergeometric functions. We give some inequalities for simple Laguerre, Laguerre, pseudo-Laguerre, Shively's pseudo-Laguerre, and Hermite functions of one and two variables.
\end{abstract}

MSC: 26D15; 30A10;26D07

Keywords: confluent hypergeometric; simple Laguerre; Laguerre; pseudo-Laguerre; Shively's pseudo-Laguerre; Hermite functions

\section{Introduction}

Inequalities for the special functions appear infrequently in the literature. Some of these inequalities are closely related to those presented here. Inequalities for the ratio of confluent hypergeometric functions are available in the literature. The formulas are very important, as they include expansions for many transcendent expressions of mathematical physics in series of the classical orthogonal polynomials, Laguerre, Hermite functions, etc. [1-8]. The developments bear heavily on the works of Luke [9-12]. The author has earlier studied the inequalities for Humbert functions [13]. This motivated us to obtain two-sided inequalities for simple Laguerre, Laguerre, pseudo-Laguerre, Shively's pseudo-Laguerre, and Hermite functions of one and two variables through confluent hypergeometric functions of one and two variables.

In [14], the confluent hypergeometric function is defined as

$$
{ }_{1} F_{1}(a ; c ; x)=\sum_{k \geq 0} \frac{(a)_{k}}{k !(c))_{k}} x^{k}, \quad c>0, x>0,
$$

where $(a)_{k}$ is the Pochhammer symbol or shifted factorial defined as

$$
(a)_{k}=a(a+1)(a+2) \cdots(a+k-1)=\frac{\Gamma(a+k)}{\Gamma(a)} ; \quad k \geq 1 ;(a)_{0}=1 .
$$

We recall here the theorems in $[15,16]$, which will be used in the investigation that follows.

Theorem 1.1 (Erdélyi et al. [17]) If $a, c>0$ and $0<x<1$, then we have the inequalities of the ratios of a confluent hypergeometric function

$$
1+\frac{a}{c} x<{ }_{1} F_{1}(a ; c ; x)<1+\frac{2 a}{c} x .
$$

(C) 2015 Shehata; licensee Springer. This article is distributed under the terms of the Creative Commons Attribution 4.0 International License (http://creativecommons.org/licenses/by/4.0/), which permits unrestricted use, distribution, and reproduction in any medium, provided you give appropriate credit to the original author(s) and the source, provide a link to the Creative Commons license, and indicate if changes were made. 
Theorem 1.2 (Bordelon [15], Ross [7]) (i) Let $c>a>0$ and $y>x>0$, then

$$
e^{x-y}<\frac{{ }_{1} F_{1}(a ; c ; x)}{{ }_{1} F_{1}(a ; c ; y)}<1
$$

(ii) Let $d>c>a>0$ and $y>x>0$, then

$$
\frac{{ }_{1} F_{1}(a ; c ; x)}{{ }_{1} F_{1}(a ; d ; y)}>\frac{\Gamma(c) \Gamma(d-a)}{\Gamma(d) \Gamma(c-a)} .
$$

Theorem 1.3 (Joshi and Bissu [16]) (i) Let $a, b, c, d>0$ and $0<x, y<1$, then

$$
\frac{1+\frac{a}{c} x}{1+\frac{2 b}{d} y}<\frac{{ }_{1} F_{1}(a ; c ; x)}{{ }_{1} F_{1}(b ; d ; y)}<\frac{1+\frac{2 a}{c} x}{1+\frac{b}{d} y}
$$

(ii) Let $c>a>0, d>b>0, x>0$ and $0<y<1$, then

$$
\frac{1-\frac{a}{c} x}{1-\frac{b}{d} y+\frac{b(b+1)}{2 d(d+1)} y^{2}}<\frac{{ }_{1} F_{1}(a ; c ;-x)}{{ }_{1} F_{1}(b ; d ;-y)}<\frac{1-\frac{a}{c} x+\frac{a(a+1)}{2 c(c+1)} x^{2}}{1-\frac{b}{d} y} .
$$

In general, a new generalized form of the confluent hypergeometric function ${ }_{2} F_{2}$ is introduced by using the integral representation method

$$
{ }_{2} F_{2}(a, b ; c, d ; x, y)=\sum_{h, k \geq 0} \frac{(a)_{h}(b)_{k}}{h ! k !(c)_{h}(d)_{k}} x^{h} y^{k}, \quad c, d>0, x, y>0 .
$$

Now, let us give a generalization for the above inequalities.

Theorem 1.4 (i) Let $a, b, c, d>0$ and $0<x, y<1$, then

$$
1+\frac{a}{c} x+\frac{b}{d} y<{ }_{2} F_{2}(a, b ; c, d ; x, y)<1+\frac{2 a}{c} x+\frac{2 b}{d} y .
$$

(ii) Let $a, a^{\prime}, b, b^{\prime}, c, c^{\prime}, d, d^{\prime}>0$ and $0<x, y, z, w<1$, then

$$
\frac{1+\frac{a}{c} x+\frac{b}{d} y}{1+\frac{2 a^{\prime}}{c^{\prime}} z+\frac{2 b^{\prime}}{d^{\prime}} w}<\frac{{ }_{2} F_{2}(a, b ; c, d ; x, y)}{{ }_{2} F_{2}\left(a^{\prime}, b^{\prime} ; c^{\prime}, d^{\prime} ; z, w\right)}<\frac{1+\frac{2 a}{c} x+\frac{2 b}{d} y}{1+\frac{a^{\prime}}{c^{\prime}} z+\frac{b^{\prime}}{d^{\prime}} w}
$$

(iii) Let $c>a>0, d>b>0, c^{\prime}>a^{\prime}>0, d^{\prime}>b^{\prime}>0, x, y>0$, and $0<z, w<1$, then

$$
\begin{aligned}
& \frac{1-\frac{a}{c} x-\frac{b}{d} y}{1-\frac{a^{\prime}}{c^{\prime}} z-\frac{b^{\prime}}{d^{\prime}} w+\frac{a^{\prime}\left(a^{\prime}+1\right)}{2 c^{\prime}\left(c^{\prime}+1\right)} z^{2}+\frac{a^{\prime} b^{\prime}}{c^{\prime} d^{\prime}} z w+\frac{b^{\prime}\left(b^{\prime}+1\right)}{2 d^{\prime}\left(d^{\prime}+1\right)} w^{2}} \\
& <\frac{{ }_{2} F_{2}(a, b ; c, d ;-x,-y)}{{ }_{2} F_{2}\left(a^{\prime}, b^{\prime} ; c^{\prime}, d^{\prime} ;-z,-w\right)}<\frac{1-\frac{a}{c} x-\frac{b}{d} y+\frac{a(a+1)}{2 c(c+1)} x^{2}+\frac{a b}{c d} x y+\frac{b(b+1)}{2 d(d+1)} y^{2}}{1-\frac{a^{\prime}}{c^{\prime}} z-\frac{b^{\prime}}{d^{\prime}} w} .
\end{aligned}
$$

\section{Inequalities for simple Laguerre functions}

The purpose of this section is to introduce new inequalities for a simple Laguerre function of one and two variables, which represents the hypergeometric functions as given by the relations (1.1)-(1.10). 
The simple Laguerre function $L_{a}(x)$ of one variable is defined by [14]

$$
L_{a}(x)={ }_{1} F_{1}(-a ; 1 ; x) .
$$

In the following some inequalities are established.

Theorem 2.1 If $a<0$ and $0<x<1$, then we have

$$
1-a x<L_{a}(x)<1-2 a x
$$

Proof Equation (2.2) follows directly from (2.1) and (1.2) by putting $c=1$.

Theorem 2.2 (i) Let $-1<a<0$ and $y>x>0$, then

$$
e^{x-y}<\frac{L_{a}(x)}{L_{a}(y)}<1
$$

(ii) If $a, b<0$ and $0<x, y<1$, then

$$
\frac{1-a x}{1-2 b y}<\frac{L_{a}(x)}{L_{b}(y)}<\frac{1-2 a x}{1-b y} .
$$

(iii) Let $-1<a, b<0, x>0$, and $0<y<1$, then

$$
\frac{1+a x}{1+b y+\frac{b(b-1)}{4} y^{2}}<\frac{L_{a}(-x)}{L_{b}(-y)}<\frac{1+a x+\frac{a(a-1)}{4} x^{2}}{1+b y} .
$$

Proof Similarly result (2.3), (2.4), and (2.5) can also be obtained with the help of (2.1), (1.3), (1.5), and (1.6).

Our interest is to show that our inequality (2.3) gives inequality at $x=0.9$ and $a=-0.5$;

$$
1.45<L_{-0.5}(0.9)<1.9 \text {. }
$$

Note also that not only the restriction that the argument is less than the order is waived, but also it holds in the extended domain $a \in(-1,0), x=0.5$, and $y=1$

$$
0.60653065971263342360379953499118<\frac{L_{-0.5}(0.5)}{L_{-0.5}(1)}<1 .
$$

As the examples for $a=-0.5,-0.25, b=-0.25,-0.5, x=0.5$, and $y=0.6$, we have from $(2.4)$

0.96153846153846153846153846153846

$$
\begin{gathered}
<\frac{L_{-0.5}(0.5)}{L_{-0.25}(0.6)}<1.3043478260869565217391304347826, \\
0.703125<\frac{L_{-0.25}(0.5)}{L_{-0.5}(0.6)}<0.96153846153846153846153846153846 .
\end{gathered}
$$


For $a=-0.5,-0.25, b=-0.25,-0.5, x=0.9$, and $y=0.5$ from (2.5), we have

0.61484716157205240174672489082969

$$
<\frac{L_{-0.5}(-0.9)}{L_{-0.25}(-0.5)}<0.80214285714285714285714285714286 \text {, }
$$

0.97254901960784313725490196078431

$$
<\frac{L_{-0.25}(-0.9)}{L_{-0.5}(-0.5)}<1.1177083333333333333333333333333 .
$$

Note also that in the common domain of validity, whereas (2.4) gives an improved lower and upper bounds, (2.5) gives only an improved lower bound.

Next, we define the simple Laguerre function of two variables by

$$
L_{a, b}(x, y)={ }_{2} F_{2}(-a, b ; 1,1 ; x, y) .
$$

Similar investigations are developed to establish new inequalities for a simple Laguerre function of two variables.

Theorem 2.3 (i) Let $a, b<0$ and $0<x, y<1$, then

$$
1-a x-b y<L_{a, b}(x, y)<1-2 a x-2 b y .
$$

(ii) If $a, b, c, d<0$ and $0<x, y, z, w<1$, then

$$
\frac{1-a x-b y}{1-2 c z-2 d w}<\frac{L_{a, b}(x, y)}{L_{c, d}(z, w)}<\frac{1-2 a x-2 b y}{1-c z-d w} .
$$

(iii) Let $-1<a, b, c, d<0, x, y>0$, and $0<z$, w $<1$, then

$$
\begin{aligned}
\frac{1+a x+b y}{1+c z+d w+\frac{c(c-1)}{4} z^{2}+c d z w+\frac{d(d-1)}{4} w^{2}} \\
<\frac{L_{a, b}(-x,-y)}{L_{c, d}(-z,-w)}<\frac{1+a x+b y+\frac{a(a-1)}{4} x^{2}+a b x y+\frac{b(b-1)}{4} y^{2}}{1+c z+d w} .
\end{aligned}
$$

Proof From (1.7), (1.8), (1.9), (1.10), and (2.6), we obtain the inequalities (2.7), (2.8), and (2.9).

The numerical computation appended below verifies these ratios under suitable restrictions, and gives bounds of ratios otherwise readily available. For the examples

$$
\begin{aligned}
1.6900 & <L_{-0.5,-0.4}(0.9,0.6)<2.3800, \\
0.7682 & <\frac{L_{-0.5,-0.4}(0.9,0.6)}{L_{-0.4,-0.7}(0.8,0.4)}<1.4875, \\
1.4948 & <\frac{L_{-0.5,-0.4}(-0.1,-0.2)}{L_{-0.4,-0.7}(-0.8,-0.4)}<2.1987 .
\end{aligned}
$$

In the next section, we discuss some inequalities for classical Laguerre functions of one and two variables. 


\section{Inequalities for classical Laguerre functions}

The classical Laguerre function $L_{a}^{(\alpha)}(x)$ of one variable of order $\alpha$ is defined as

$$
L_{a}^{(\alpha)}(x)=\frac{(1+\alpha)_{a}}{\Gamma(a+1)}{ }_{1} F_{1}(-a ; 1+\alpha ; x)
$$

Proceeding in a similar manner we can derive bounds for classical Laguerre function of one variable, but details are omitted.

Theorem 3.1 For $a<0, \alpha>-1$, and $0<x<1$, we have

$$
\left(1-\frac{a}{\alpha+1} x\right)\left(\frac{(1+\alpha)_{a}}{\Gamma(a+1)}\right)<L_{a}^{(\alpha)}(x)<\left(1-\frac{2 a}{\alpha+1} x\right)\left(\frac{(1+\alpha)_{a}}{\Gamma(a+1)}\right)
$$

Proof This can be proved by using (1.2) and (3.1).

Theorem 3.2 (i) If $\alpha+1>-a>0$ and $y>x>0$, then

$$
e^{x-y}<\frac{L_{a}^{(\alpha)}(x)}{L_{a}^{(\alpha)}(y)}<1
$$

(ii) If $\beta+1>\alpha+1>-a>0$, then

$$
\frac{L_{a}^{(\alpha)}(x)}{L_{a}^{(\beta)}(x)}>1
$$

(iii) If $a, b<0$ and $0<x, y<1$, then

$$
\begin{aligned}
&\left(\frac{1-\frac{a}{\alpha+1} x}{1-\frac{2 b}{\beta+1} y}\right)\left(\frac{\Gamma(b+1)(1+\alpha)_{a}}{\Gamma(a+1)(1+\beta)_{b}}\right) \\
&<\frac{L_{a}^{(\alpha)}(x)}{L_{b}^{(\beta)}(y)}<\left(\frac{1-\frac{2 a}{\alpha+1} x}{1-\frac{b}{\beta+1} y}\right)\left(\frac{\Gamma(b+1)(1+\alpha)_{a}}{\Gamma(a+1)(1+\beta)_{b}}\right) .
\end{aligned}
$$

(iv) If $\alpha+1>-a>0, \beta+1>-b>0, x>0$ and $0<y<1$, then

$$
\begin{aligned}
& \left(\frac{1+\frac{a}{\alpha+1} x}{1+\frac{b}{\beta+1} y+\frac{b(b-1)}{2(\beta+1)(\beta+2)} y^{2}}\right)\left(\frac{\Gamma(b+1)(1+\alpha)_{a}}{\Gamma(a+1)(1+\beta)_{b}}\right) \\
& \quad<\frac{L_{a}^{(\alpha)}(-x)}{L_{b}^{(\beta)}(-y)}<\left(\frac{1+\frac{a}{\alpha+1} x+\frac{a(a-1)}{2(\alpha+1)(\alpha+2)} x^{2}}{1+\frac{b}{\beta+1} y}\right)\left(\frac{\Gamma(b+1)(1+\alpha)_{a}}{\Gamma(a+1)(1+\beta)_{b}}\right) .
\end{aligned}
$$

Proof The proof of the theorem is similar to the proof of Theorem 2.2.

For the set of values $x=0.5$ and $y=1$, we have from the inequalities (3.2) and (3.3)

$$
\begin{aligned}
& 1.25<L_{-0.5}^{(0)}(0.5)<1.5 \\
& 0.74272306776217823358812422907173 \\
& \quad<L_{-0.5}^{(0.5)}(0.5)<0.84882636315677512410071340465341 .
\end{aligned}
$$


Some special cases of inequalities for classical Laguerre function are listed below:

1.4112903225806451612903225806452

$$
<\frac{L_{-0.5}^{(0.5)}(0.5)}{L_{-0.5}^{(1.5)}(0.6)}<1.7857142857142857142857142857143,
$$

0.52380952380952380952380952380952

$$
<\frac{L_{-0.5}^{(1.5)}(0.5)}{L_{-0.5}^{(0.5)}(0.6)}<0.66666666666666666666666666666667 \text {, }
$$

$1.5<\frac{L_{-0.5}^{(0.5)}(0.6)}{L_{-0.5}^{(1.5)}(0.5)}<1.9090909090909090909090909090909$,

and

$$
0.56<\frac{L_{-0.5}^{(1.5)}(0.6)}{L_{-0.5}^{(0.5)}(0.5)}<0.70857142857142857142857142857143 .
$$

The previous properties can be generalized as follows: We define the classical Laguerre function $L_{a, b}^{(\alpha, \beta)}(x, y)$ of two variables in the form

$$
L_{a, b}^{(\alpha, \beta)}(x, y)=\frac{(1+\alpha)_{a}(1+\beta)_{b}}{\Gamma(a+1) \Gamma(b+1)}{ }_{2} F_{2}(-a,-b ; 1+\alpha, 1+\beta ; x, y) .
$$

Now, let us give a generalization for the above inequalities.

Theorem 3.3 (i) Let $a, b<0, \alpha, \beta>-1$, and $0<x, y<1$, then

$$
\begin{aligned}
(1 & \left.-\frac{a}{1+\alpha} x-\frac{b}{1+\beta} y\right)\left(\frac{(1+\alpha)_{a}(1+\beta)_{b}}{\Gamma(a+1) \Gamma(b+1)}\right) \\
& <L_{a, b}^{(\alpha, \beta)}(x, y)<\left(1-\frac{2 a}{1+\alpha} x-\frac{2 b}{1+\beta} y\right)\left(\frac{(1+\alpha)_{a}(1+\beta)_{b}}{\Gamma(a+1) \Gamma(b+1)}\right) .
\end{aligned}
$$

(ii) Let $a, b, c, d<0$ and $0<x, y, z, w<1$, then

$$
\begin{aligned}
\left(\frac{1-\frac{a}{1+\alpha} x-\frac{b}{1+\beta} y}{1-\frac{2 c}{\mu+1} z-\frac{2 d}{v+1} w}\right)\left(\frac{\Gamma(c+1) \Gamma(d+1)(1+\alpha)_{a}(1+\beta)_{b}}{\Gamma(a+1) \Gamma(b+1)(1+\mu)_{c}(1+v)_{d}}\right) \\
\quad<\frac{L_{a, b}^{(\alpha, \beta)}(x, y)}{L_{c, d}^{(\mu, v)}(z, w)}<\left(\frac{1-\frac{2 a}{\alpha+1} x-\frac{2 b}{1+\beta} y}{1-\frac{c}{1+\mu} z-\frac{d}{1+\nu} w}\right)\left(\frac{\Gamma(c+1) \Gamma(d+1)(1+\alpha)_{a}(1+\beta)_{b}}{\Gamma(a+1) \Gamma(b+1)(1+\mu)_{c}(1+\nu)_{d}}\right) .
\end{aligned}
$$

(iii) Let $\alpha+1>-a>0, \beta+1>-b>0, \mu+1>-c>0, v+1>-d>0, x, y>0$, and $0<z, w<1$, then

$$
\begin{aligned}
& \left(\frac{1+\frac{a}{\alpha+1} x+\frac{b}{\beta+1} y}{1+\frac{c}{\mu+1} z+\frac{d}{v+1} w+\frac{c(c-1)}{2(\mu+1)(\mu+2)} z^{2}+\frac{c d}{(\mu+1)(v+1)} z w+\frac{d(d-1)}{2(v+1)(v+2)} w^{2}}\right) \\
& \quad \times\left(\frac{\Gamma(c+1) \Gamma(d+1)(1+\alpha)_{a}(1+\beta)_{b}}{\Gamma(a+1) \Gamma(b+1)(1+\mu)_{c}(1+v)_{d}}\right)
\end{aligned}
$$




$$
\begin{aligned}
< & \frac{L_{a, b}^{(\alpha, \beta)}(-x,-y)}{L_{c, d}^{(\mu, v)}(-z,-w)}<\left(\frac{1+\frac{a}{\alpha+1} x+\frac{b}{\beta+1} y+\frac{a(a-1)}{2(\alpha+1)(\alpha+2)} x^{2}+\frac{a b}{(\alpha+1)(\beta+1)} x y+\frac{b(b-1)}{2(\beta+1)(\beta+2)} y^{2}}{1+\frac{c}{\mu+1} z+\frac{d}{v+1} w}\right) \\
& \times\left(\frac{\Gamma(c+1) \Gamma(d+1)(1+\alpha)_{a}(1+\beta)_{b}}{\Gamma(a+1) \Gamma(b+1)(1+\mu)_{c}(1+v)_{d}}\right) .
\end{aligned}
$$

Proof The proof of the theorem is very similar to Theorem 2.3.

In particular, for the set of values $\alpha=1,0.5,0.7, \beta=2,0.7,0.6, x=0.9,0.8$, and $y=$ $0.6,0.4$, we have from (3.8), (3.9), and (3.10), respectively, the inequalities:

$$
\begin{aligned}
& 0.3132<L_{-0.5,-0.4}^{(1,2)}(0.9,0.6)<0.3864, \quad 0.6085<L_{-0.5,-0.4}^{(0.5,0.7)}(0.9,0.6)<0.7948, \\
& 0.6861<\frac{L_{-0.5,-0.4}^{(0.5,0.7)}(0.9,0.6)}{L_{-0.3,-0.2}^{(0.7,0.6)}(0.8,0.4)}<1.0399 .
\end{aligned}
$$

In the next section, we discuss some inequalities for pseudo-Laguerre functions for different values that exhibit very interesting behavior of these function.

\section{Inequalities for pseudo-Laguerre functions}

For the pseudo-Laguerre function of one variable $f_{a}(x ; \lambda)[14,18]$ defined by

$$
f_{a}(x ; \lambda)=\frac{(-\lambda)_{a}}{\Gamma(a+1)}{ }_{1} F_{1}(-a ; 1+\lambda-a ; x)
$$

where $\lambda$ is non-integral, the following inequalities for pseudo-Laguerre function are established.

Theorem 4.1 For the pseudo-Laguerre functions $f_{a}(x ; \lambda)$ we have the inequalities:

$$
\begin{aligned}
& \left(1-\frac{a}{1+\lambda-a} x\right)\left(\frac{(-\lambda)_{a}}{\Gamma(a+1)}\right)<f_{a}(x ; \lambda)<\left(1-\frac{2 a}{1+\lambda-a} x\right)\left(\frac{(-\lambda)_{a}}{\Gamma(a+1)}\right) \\
& \quad a<0,0<x<1, \\
& e^{x-y}<\frac{f_{a}(x ; \lambda)}{f_{a}(y ; \lambda)}<1 ; \quad 1+\lambda-a>-a>0, y>x>0, \\
& \frac{f_{a}(x ; \lambda)}{f_{a}(y ; \mu)}>\frac{(-\lambda)_{a}}{(-\mu)_{a}} \frac{\Gamma(1+\lambda-a) \Gamma(1+\mu)}{\Gamma(1+\mu-a) \Gamma(1+\lambda)} ; \quad 1+\mu-a>1+\lambda-a>-a>0, y>x>0, \\
& \left(\frac{1-\frac{a}{1+\lambda-a} x}{1-\frac{2 b}{1+\mu-b} y}\right)\left(\frac{\Gamma(b+1)(-\lambda)_{a}}{\Gamma(a+1)(-\mu)_{b}}\right)<\frac{f_{a}(x ; \lambda)}{f_{b}(y ; \mu)}<\left(\frac{1-\frac{2 a}{1+\lambda-a} x}{1-\frac{b}{1+\mu-b} y}\right)\left(\frac{\Gamma(b+1)(-\lambda)_{a}}{\Gamma(a+1)(-\mu)_{b}}\right) \\
& \quad a, b<0,0<x, y<1
\end{aligned}
$$

and

$$
\begin{aligned}
& \left(\frac{1+\frac{a}{\lambda-a+1} x}{1+\frac{b}{1+\mu-b} y+\frac{b(b-1)}{2(\mu-b+1)(\mu-b+2)} y^{2}}\right)\left(\frac{\Gamma(b+1)(-\lambda)_{a}}{\Gamma(a+1)(-\mu)_{b}}\right) \\
& <\frac{f_{a}(-x ; \lambda)}{f_{b}(-y ; \mu)}<\left(\frac{1+\frac{a}{\lambda-a+1} x+\frac{a(a-1)}{2(\lambda-a+1)(\lambda-a+2)} x^{2}}{1+\frac{b}{1+\mu-b} y}\right)\left(\frac{\Gamma(b+1)(-\lambda)_{a}}{\Gamma(a+1)(-\mu)_{b}}\right) ; \\
& \lambda-a+1>-a>0,1+\mu-b>-b>0, x>0,0<y<1 .
\end{aligned}
$$


Proof From (1.1)-(1.6) and (4.1), we obtain the inequalities for pseudo-Laguerre function (4.2)-(4.6).

From (4.3) when $x=0.05,0.5,9$ and $y=0.06,0.6,10$, we get

$$
\begin{aligned}
0.990049833 & <\frac{f_{a}(0.05 ; \lambda)}{f_{a}(0.06 ; \lambda)}<1, \\
0.904837418 & <\frac{f_{a}(0.5 ; \lambda)}{f_{a}(0.6 ; \lambda)}<1, \\
0.367879441 & <\frac{f_{a}(9 ; \lambda)}{f_{a}(10 ; \lambda)}<1 .
\end{aligned}
$$

Let $\lambda$ and $\mu$ be non-integral, we define the pseudo-Laguerre function $f_{a, b}(x, y ; \lambda, \mu)$ of two variables in the form

$$
f_{a, b}(x, y ; \lambda, \mu)=\frac{(-\lambda)_{a}(-\mu)_{b}}{\Gamma(a+1) \Gamma(b+1)}{ }_{2} F_{2}(-a,-b ; 1+\lambda-a, 1+\mu-b ; x, y) .
$$

Next, we discuss further the pseudo-Laguerre function of two variables and the inequalities and properties investigated.

Theorem 4.2 (i) If $a, b<0$ and $0<x, y<1$, then

$$
\begin{aligned}
(1 & \left.-\frac{a}{1+\lambda-a} x-\frac{b}{1+\mu-b} y\right)\left(\frac{(-\lambda)_{a}(-\mu)_{b}}{\Gamma(a+1) \Gamma(b+1)}\right) \\
& <f_{a, b}(x, y ; \lambda, \mu)<\left(1-\frac{2 a}{1+\lambda-a} x-\frac{2 b}{1+\mu-b} y\right)\left(\frac{(-\lambda)_{a}(-\mu)_{b}}{\Gamma(a+1) \Gamma(b+1)}\right) .
\end{aligned}
$$

(ii) If $a, b, c, d<0$ and $0<x, y, z, w<1$, then

$$
\begin{aligned}
& \left(\frac{1-\frac{a}{1+\lambda-a} x-\frac{b}{1+\mu-b} y}{1-\frac{2 c}{1+\nu-c} z-\frac{2 d}{1+\xi-d} w}\right)\left(\frac{\Gamma(c+1) \Gamma(d+1)(-\lambda)_{a}(-\mu)_{b}}{\Gamma(a+1) \Gamma(b+1)(-v)_{c}(-\xi)_{d}}\right) \\
& <\frac{f_{a, b}(x, y ; \lambda, \mu)}{f_{c, d}(z, w ; v, \xi)}<\left(\frac{1-\frac{2 a}{1+\lambda-a} x-\frac{2 b}{1+\mu-b} y}{1-\frac{c}{1+v-c} z-\frac{d}{1+\xi-d} w}\right)\left(\frac{\Gamma(c+1) \Gamma(d+1)(-\lambda)_{a}(-\mu)_{b}}{\Gamma(a+1) \Gamma(b+1)(-v)_{c}(-\xi)_{d}}\right) .
\end{aligned}
$$

(iii) If $\lambda-a+1>-a>0,1+\mu-b>-b>0,1+v-c>-c>0,1+\xi-d>-d>0, x, y>0$, and $0<z, w<1$, then

$$
\begin{aligned}
& \left(\frac{1+\frac{a}{\lambda-a+1} x+\frac{b}{\mu-b+1} y}{1+\frac{c}{1+\nu-c} z+\frac{d}{1+\xi-d} w+\frac{c(c-1)}{2(v-c+1)(v-c+2)} z^{2}+\frac{c d}{(v-c+1)(\xi-d+1)} z w+\frac{d(d-1)}{2(\xi-d+1)(\xi-d+2)} w^{2}}\right) \\
& \quad \times\left(\frac{\Gamma(c+1) \Gamma(d+1)(-\lambda)_{a}(-\mu)_{b}}{\Gamma(a+1) \Gamma(b+1)(-v)_{c}(-\xi)_{d}}\right) \\
& <\frac{f_{a, b}(-x,-y ; \lambda, \mu)}{f_{c, d}(-z,-w ; \nu, \xi)} \\
& <\left(\frac{1+\frac{a}{\lambda-a+1} x+\frac{b}{\mu-b+1} y+\frac{a(a-1)}{2(\lambda-a+1)(\lambda-a+2)} x^{2}+\frac{a b}{(\lambda-a+1)(\mu-b+1)} x y+\frac{b(b-1)}{2(\mu-b+1)(\mu-b+2)} y^{2}}{1+\frac{c}{1+\nu-c} z+\frac{d}{1+\xi-d} w}\right) \\
& \quad \times\left(\frac{\Gamma(c+1) \Gamma(d+1)(-\lambda)_{a}(-\mu)_{b}}{\Gamma(a+1) \Gamma(b+1)(-v)_{c}(-\xi)_{d}}\right) .
\end{aligned}
$$


Proof By using (1.7)-(1.10) and (4.7), we obtain the inequalities (4.8)-(4.10).

Numerically, Theorem 4.2 gives

$$
\begin{aligned}
& 1.0800<f_{-0.5,-0.4}(0.7,0.9 ; 0.3,0.4)<1.3855, \\
& 1.0714<f_{-0.5,-0.4}(0.9,0.6 ; 0.3,0.4)<1.3683 .
\end{aligned}
$$

In the next section, we show that there exist operational relations between confluent hypergeometric functions and the various types of Shively's pseudo-Laguerre functions.

\section{Inequalities for Shively's pseudo-Laguerre functions}

Shively [18] studied the pseudo-Laguerre function of one variable by

$$
R_{a}(\alpha, x)=\frac{(\alpha)_{2 a}}{\Gamma(a+1)(\alpha)_{a}}{ }_{1} F_{1}(-a ; \alpha+a ; x) .
$$

By using inequalities of a confluent hypergeometric function, we can obtain some inequalities for Shively's pseudo-Laguerre function in the following theorem.

\section{Theorem 5.1}

$$
\begin{aligned}
& \left(1-\frac{a}{\alpha+a} x\right)\left(\frac{(\alpha)_{2 a}}{\Gamma(a+1)(\alpha)_{a}}\right)<R_{a}(\alpha, x)<\left(1-\frac{2 a}{\alpha+a} x\right)\left(\frac{(\alpha)_{2 a}}{\Gamma(a+1)(\alpha)_{a}}\right) \\
& \quad a<0, \alpha+a>0,0<x<1, \\
& e^{x-y}<\frac{R_{a}(\alpha, x)}{R_{a}(\alpha, y)}<1 ; \quad \alpha+a>-a>0, y>x>0, \\
& \frac{R_{a}(\alpha, x)}{R_{a}(\beta, y)}>\frac{(\alpha)_{2 a}(\beta)_{a}}{(\alpha)_{a}(\beta)_{2 a}} \frac{\Gamma(\alpha+a) \Gamma(\beta+2 a)}{\Gamma(\beta+a) \Gamma(\alpha+2 a)} ; \quad \beta+a>\alpha+a>-a>0, \\
& \left(\frac{1-\frac{a}{\alpha+x} x}{1-\frac{2 b}{\beta+b} y}\right)\left(\frac{\Gamma(b+1)(\alpha)_{2 a}(\beta)_{b}}{\Gamma(a+1)(\alpha)_{a}(\beta)_{2 b}}\right)<\frac{R_{a}(\alpha, x)}{R_{b}(\beta, y)}<\left(\frac{1-\frac{2 a}{\alpha+a} x}{1-\frac{b}{\beta+b} y}\right)\left(\frac{\Gamma(b+1)(\alpha)_{2 a}(\beta)_{b}}{\Gamma(a+1)(\alpha)_{a}(\beta)_{2 b}}\right) ; \\
& \quad a, b<0, \alpha+a, \beta+b>0,0<x, y<1
\end{aligned}
$$

and

$$
\begin{aligned}
& \left(\frac{1+\frac{a}{\alpha+a} x}{1+\frac{b}{\beta+b} y+\frac{b(b-1)}{2(\beta+b)(\beta+b+1)} y^{2}}\right)\left(\frac{\Gamma(b+1)(\alpha)_{2 a}(\beta)_{b}}{\Gamma(a+1)(\alpha)_{a}(\beta)_{2 b}}\right) \\
& <\frac{R_{a}(\alpha,-x)}{R_{b}(\beta,-y)}<\left(\frac{1+\frac{a}{\alpha+a} x+\frac{a(a-1)}{2(\alpha+a)(\alpha+a+1)} x^{2}}{1+\frac{b}{\beta+b} y}\right)\left(\frac{\Gamma(b+1)(\alpha)_{2 a}(\beta)_{b}}{\Gamma(a+1)(\alpha)_{a}(\beta)_{2 b}}\right) ; \\
& \alpha+a>-a>0, \beta+b>-b>0, x>0,0<y<1 .
\end{aligned}
$$

Proof This can be proved by using (1.2)-(1.6) and (5.1).

In particular, for $a=-0.5, \alpha=2,3$ and $x=0.5$, we have from (5.2) the inequalities

$$
\begin{aligned}
& 0.742723067<R_{-0.5}(2,0.5)<0.848826363, \\
& 0.466854499<R_{-0.5}(3,0.5)<0.509295817 .
\end{aligned}
$$


Next, we define the Shively's pseudo-Laguerre function of two variables as

$$
R_{a, b}(\alpha, \beta, x, y)=\frac{(\alpha)_{2 a}(\beta)_{2 b}}{\Gamma(a+1) \Gamma(b+1)(\alpha)_{a}(\beta)_{b}}{ }_{2} F_{2}(-a,-b ; \alpha+a, \beta+b ; x, y)
$$

A generalization for the above inequalities is given as follows.

Theorem 5.2 (i) If $a, b<0, \alpha+2 a>0, \beta+2 b>0$, and $0<x, y<1$, then

$$
\begin{aligned}
& \left(1-\frac{a}{\alpha+a} x-\frac{b}{\beta+b} y\right)\left(\frac{(\alpha)_{2 a}(\beta)_{2 b}}{\Gamma(a+1) \Gamma(b+1)(\alpha)_{a}(\beta)_{b}}\right) \\
& \quad<R_{a, b}(\alpha, \beta, x, y)<\left(1-\frac{2 a}{\alpha+a} x-\frac{2 b}{\beta+b} y\right)\left(\frac{(\alpha)_{2 a}(\beta)_{2 b}}{\Gamma(a+1) \Gamma(b+1)(\alpha)_{a}(\beta)_{b}}\right) .
\end{aligned}
$$

(ii) If $a, b, c, d<0, \alpha+2 a, \beta+2 b, \mu+2 c, v+2 d>0$, and $0<x, y, z, w<1$, then

$$
\begin{aligned}
& \left(\frac{1-\frac{a}{\alpha+a} x-\frac{b}{\beta+b} y}{1-\frac{2 c}{\mu+c} z-\frac{2 d}{v+d} w}\right)\left(\frac{\Gamma(c+1) \Gamma(d+1)(\alpha)_{2 a}(\beta)_{2 b}(\mu)_{c}(v)_{d}}{\Gamma(a+1) \Gamma(b+1)(\alpha)_{a}(\beta)_{b}(\mu)_{2 c}(\nu)_{2 d}}\right) \\
& <\frac{R_{a, b}(\alpha, \beta, x, y)}{R_{c, d}(\mu, \nu, z, w)} \\
& <\left(\frac{1-\frac{2 a}{\alpha+a} x-\frac{2 b}{\beta+b} y}{1-\frac{c}{\mu+c} z-\frac{d}{v+d} w}\right)\left(\frac{\Gamma(c+1) \Gamma(d+1)(\alpha)_{2 a}(\beta)_{2 b}(\mu)_{c}(\nu)_{d}}{\Gamma(a+1) \Gamma(b+1)(\alpha)_{a}(\beta)_{b}(\mu)_{2 c}(\nu)_{2 d}}\right) .
\end{aligned}
$$

(iii) If $\alpha+a>-a>0, \beta+b>-b>0, \mu+c>-c>0, v+d>-d>0, x, y>0$, and $0<z, w<1$, then

$$
\begin{aligned}
& \left(\frac{1+\frac{a}{\alpha+a} x+\frac{b}{\beta+b} y}{1+\frac{c}{\mu+c} z+\frac{d}{v+d} w+\frac{c(c-1)}{2(\mu+c)(\mu+c+1)} z^{2}+\frac{c d}{(\mu+c)(v+d)} z w+\frac{d(d-1)}{2(v+d)(v+d+1)} w^{2}}\right) \\
& \quad \times\left(\frac{\Gamma(c+1) \Gamma(d+1)(\alpha)_{2 a}(\beta)_{2 b}(\mu)_{c}(\nu)_{d}}{\Gamma(a+1) \Gamma(b+1)(\alpha)_{a}(\beta)_{b}(\mu)_{2 c}(\nu)_{2 d}}\right) \\
& <\frac{R_{a, b}(\alpha, \beta,-x,-y)}{R_{c, d}(\mu, v,-z,-w)} \\
& <\left(\frac{1+\frac{a}{\alpha+a} x+\frac{b}{\beta+b} y+\frac{a(a-1)}{2(\alpha+a)(\alpha+a+1)} x^{2}+\frac{a b}{(\alpha+a)(\beta+a)} x y+\frac{b(b-1)}{2(\beta+b)(\beta+b+1)} y^{2}}{1+\frac{c}{\mu+c} z+\frac{d}{v+d} w}\right) \\
& \quad \times\left(\frac{\Gamma(c+1) \Gamma(d+1)(\alpha)_{2 a}(\beta)_{2 b}(\mu)_{c}(v)_{d}}{\Gamma(a+1) \Gamma(b+1)(\alpha)_{a}(\beta)_{b}(\mu)_{2 c}(v)_{2 d}}\right) .
\end{aligned}
$$

Proof Using the inequalities of confluent hypergeometric function (1.7)-(1.10) and (5.5), we obtain the inequalities for Shively's pseudo-Laguerre function.

As a numerical verification, we have from Theorem 5.2:

$$
\begin{aligned}
& 1.1693<R_{-0.5,-0.3}(2,1,0.8,0.3)<1.5006, \\
& 1.4331<R_{-0.5,-0.5}(1.5,1.6,0.7,0.6)<1.9831, \\
& 1.2242<R_{-1.5,-1.3}(3.5,2.8,0.7,0.6)<1.8497, \\
& 5.4152<R_{-0.7,-0.9}(1.5,1.9,0.3,0.8)<8.0989 .
\end{aligned}
$$


In the next section, we discuss further the various types of Hermite functions and the inequalities and properties investigated.

\section{Inequalities for Hermite functions}

The Hermite function of one variable $H_{2 a}(x)$ is defined by

$$
H_{2 a}(x)=\frac{(-1)^{a} \Gamma(2 a+1)}{\Gamma(a+1)}{ }_{1} F_{1}\left(-a ; \frac{1}{2} ; x^{2}\right) .
$$

The above arguments establish the inequalities for a Hermite function $H_{2 a}(x)$ in the following theorem.

Theorem 6.1 (i) If $a<0$ and $0<x^{2}<1$, then

$$
\left(1-2 a x^{2}\right)\left(\frac{(-1)^{a} \Gamma(2 a+1)}{\Gamma(a+1)}\right)<H_{2 a}(x)<\left(1-4 a x^{2}\right)\left(\frac{(-1)^{a} \Gamma(2 a+1)}{\Gamma(a+1)}\right) .
$$

(ii) If $-\frac{1}{2}<a<0$ and $y^{2}>x^{2}>0$, then

$$
e^{x^{2}-y^{2}}<\frac{H_{2 a}(x)}{H_{2 a}(y)}<1
$$

(iii) If $a, b<0$ and $0<x^{2}, y^{2}<1$, then

$$
\begin{aligned}
&\left(\frac{1-2 a x^{2}}{1-4 b y^{2}}\right)\left(\frac{(-1)^{a} \Gamma(2 a+1) \Gamma(b+1)}{(-1)^{b} \Gamma(a+1) \Gamma(2 b+1)}\right) \\
&<\frac{H_{2 a}(x)}{H_{2 b}(y)}<\left(\frac{1-4 a x^{2}}{1-2 b y^{2}}\right)\left(\frac{(-1)^{a} \Gamma(2 a+1) \Gamma(b+1)}{(-1)^{b} \Gamma(a+1) \Gamma(2 b+1)}\right) .
\end{aligned}
$$

(iv) If $-\frac{1}{2}<a, b<0$ and $x^{2}>0,0<y^{2}<1$, then

$$
\begin{aligned}
& \left(\frac{1+2 a x^{2}}{1+2 b y^{2}+\frac{2}{3} b(b-1) y^{4}}\right)\left(\frac{(-1)^{a} \Gamma(2 a+1) \Gamma(b+1)}{(-1)^{b} \Gamma(a+1) \Gamma(2 b+1)}\right) \\
& \quad<\frac{H_{2 a}(x)}{H_{2 b}(y)}<\left(\frac{1+2 a x^{2}+\frac{2}{3} a(a-1) x^{4}}{1+2 b y^{2}}\right)\left(\frac{(-1)^{a} \Gamma(2 a+1) \Gamma(b+1)}{(-1)^{b} \Gamma(a+1) \Gamma(2 b+1)}\right) .
\end{aligned}
$$

It has given the following form for the operational relations of this Hermite function of one variable $H_{2 a+1}(x)$ [13]:

$$
H_{2 a+1}(x)=\frac{(-1)^{a} 2 \Gamma(2 a+2)}{\Gamma(a+1)} x_{1} F_{1}\left(-a ; \frac{3}{2} ; x^{2}\right)
$$

Using inequalities of confluent hypergeometric functions appropriately, we obtain some rather complicated estimates.

\section{Theorem 6.2}

$$
\begin{aligned}
& \left(1-\frac{2}{3} a x^{2}\right)\left(\frac{(-1)^{a} 2 \Gamma(2 a+2) x}{\Gamma(a+1)}\right)<H_{2 a+1}(x)<\left(1-\frac{4}{3} a x^{2}\right)\left(\frac{(-1)^{a} 2 \Gamma(2 a+2) x}{\Gamma(a+1)}\right) \\
& a<0,0<x^{2}<1
\end{aligned}
$$




$$
\begin{aligned}
& e^{x^{2}-y^{2}}\left(\frac{x}{y}\right)<\frac{H_{2 a+1}(x)}{H_{2 a+1}(y)}<\left(\frac{x}{y}\right), \quad-\frac{3}{2}<a<0, y^{2}>x^{2}>0, \\
& \frac{H_{2 a}(x)}{H_{2 a+1}(y)}>\frac{(1-2 a)}{2(2 a+1) y}, \quad-\frac{3}{2}<a<0, \\
& \left(\frac{1-\frac{2}{3} a x^{2}}{1-\frac{4}{3} b y^{2}}\right)\left(\frac{(-1)^{a} \Gamma(b+1) \Gamma(2 a+2) x}{(-1)^{b} \Gamma(a+1) \Gamma(2 b+2) y}\right) \\
& \quad<\frac{H_{2 a+1}(x)}{H_{2 b+1}(y)}<\left(\frac{1-\frac{4}{3} a x^{2}}{1-\frac{2}{3} b y^{2}}\right)\left(\frac{(-1)^{a} \Gamma(b+1) \Gamma(2 a+2) x}{(-1)^{b} \Gamma(a+1) \Gamma(2 b+2) y}\right) ; \\
& a, b<0,0<x^{2}, y^{2}<1
\end{aligned}
$$

and

$$
\begin{aligned}
& \left(\frac{1+\frac{2}{3} a x^{2}}{1+\frac{2}{3} b y^{2}+\frac{2 b(b-1)}{15} y^{4}}\right)\left(\frac{(-1)^{a} \Gamma(b+1) \Gamma(2 a+2) x}{(-1)^{b} \Gamma(a+1) \Gamma(2 b+2) y}\right) \\
& <\frac{H_{2 a+1}(-x)}{H_{2 b+1}(-y)}<\left(\frac{1+\frac{2}{3} a x^{2}+\frac{2 a(a-1)}{15} x^{4}}{1+\frac{2}{3} b y^{2}}\right)\left(\frac{(-1)^{a} \Gamma(b+1) \Gamma(2 a+2) x}{(-1)^{b} \Gamma(a+1) \Gamma(2 b+2) y}\right) \\
& \quad-\frac{3}{2}<a, b<0, x^{2}>0,0<y^{2}<1 .
\end{aligned}
$$

Proof From (1.2)-(1.6) and (6.6), it is easy to see that (6.7)-(6.11) hold.

For example, when $a=-0.25, x=0.25$ and $y=0.5$, from (6.3) we get

$$
0.829029118<\frac{H_{-0.5}(0.25)}{H_{-0.5}(0.5)}<1 .
$$

The previous results can be generalized as follows. We define the Hermite function $H_{2 a, 2 b}(x, y)$ of two variables by the relation

$$
H_{2 a, 2 b}(x, y)=\frac{(-1)^{a+b} \Gamma(2 a+1) \Gamma(2 b+1)}{\Gamma(a+1) \Gamma(b+1)}{ }_{2} F_{2}\left(-a,-b ; \frac{1}{2}, \frac{1}{2} ; x^{2}, y^{2}\right) .
$$

A generalization for the above inequalities is given in the following.

Theorem 6.3 (i) Let $a, b<0$ and $0<x^{2}, y^{2}<1$, then

$$
\begin{aligned}
(1 & \left.-2 a x^{2}-2 b y^{2}\right)\left(\frac{(-1)^{a+b} \Gamma(2 a+1) \Gamma(2 b+1)}{\Gamma(a+1) \Gamma(b+1)}\right) \\
<H_{2 a, 2 b}(x, y) & <\left(1-4 a x^{2}-4 b y^{2}\right)\left(\frac{(-1)^{a+b} \Gamma(2 a+1) \Gamma(2 b+1)}{\Gamma(a+1) \Gamma(b+1)}\right) .
\end{aligned}
$$

(ii) Let $a, b, c, d<0$ and $0<x^{2}, y^{2}, z^{2}, w^{2}<1$, then

$$
\begin{aligned}
& \left(\frac{1-2 a x^{2}-2 b y^{2}}{1-4 c z^{2}-4 d w^{2}}\right)\left(\frac{(-1)^{a+b} \Gamma(2 a+1) \Gamma(2 b+1) \Gamma(c+1) \Gamma(d+1)}{(-1)^{c+d} \Gamma(a+1) \Gamma(b+1) \Gamma(2 c+1) \Gamma(2 d+1)}\right) \\
& \quad<\frac{H_{2 a, 2 b}(x, y)}{H_{2 c, 2 d}(z, w)}<\left(\frac{1-4 a x^{2}-4 b y^{2}}{1-2 c z^{2}-2 d w^{2}}\right)\left(\frac{(-1)^{a+b} \Gamma(2 a+1) \Gamma(2 b+1) \Gamma(c+1) \Gamma(d+1)}{(-1)^{c+d} \Gamma(a+1) \Gamma(b+1) \Gamma(2 c+1) \Gamma(2 d+1)}\right) .
\end{aligned}
$$


(iii) Let $-\frac{1}{2}<a, b, c, d<0, x^{2}, y^{2}>0$, and $0<z^{2}, w^{2}<1$, then

$$
\begin{aligned}
& \left(\frac{1+2 a x^{2}+2 b y^{2}}{1+2 c z^{2}+2 d w^{2}+\frac{2}{3} c(c-1) z^{4}+4 c d z^{2} w^{2}+\frac{2}{3} d(d-1) w^{4}}\right) \\
& \times\left(\frac{(-1)^{a+b} \Gamma(2 a+1) \Gamma(2 b+1) \Gamma(c+1) \Gamma(d+1)}{(-1)^{c+d} \Gamma(a+1) \Gamma(b+1) \Gamma(2 c+1) \Gamma(2 d+1)}\right) \\
& <\frac{H_{2 a, 2 b}(x, y)}{H_{2 c, 2 d}(z, w)}<\left(\frac{1+2 a x^{2}+2 b y^{2}+\frac{2}{3} a(a-1) x^{4}+4 a b x^{2} y^{2}+\frac{2}{3} b(b-1) y^{4}}{1+2 c z^{2}+2 d w^{2}}\right) \\
& \times\left(\frac{(-1)^{a+b} \Gamma(2 a+1) \Gamma(2 b+1) \Gamma(c+1) \Gamma(d+1)}{(-1)^{c+d} \Gamma(a+1) \Gamma(b+1) \Gamma(2 c+1) \Gamma(2 d+1)}\right) .
\end{aligned}
$$

Proof From (1.7)-(1.10) and (6.12), it is easy to see that (6.13)-(6.15) hold.

Secondly, we define the Hermite function $H_{2 a+1,2 b+1}(x, y)$ of two variables by the confluent hypergeometric functions as in the form

$$
H_{2 a+1,2 b+1}(x, y)=\frac{(-1)^{a+b} 4 \Gamma(2 a+2) \Gamma(2 b+2)}{\Gamma(a+1) \Gamma(b+1)} x y_{2} F_{2}\left(-a,-b ; \frac{3}{2}, \frac{3}{2} ; x^{2}, y^{2}\right) .
$$

Now, let us give a generalization for the above inequalities.

Theorem 6.4 (i) If $a, b<0$ and $0<x^{2}, y^{2}<1$, then

$$
\begin{aligned}
(1 & \left.-\frac{2}{3} a x^{2}-\frac{2}{3} b y^{2}\right)\left(\frac{(-1)^{a+b} 4 \Gamma(2 a+2) \Gamma(2 b+2) x y}{\Gamma(a+1) \Gamma(b+1)}\right) \\
& <H_{2 a+1,2 b+1}(x, y)<\left(1-\frac{4}{3} a x^{2}-\frac{4}{3} b y^{2}\right)\left(\frac{(-1)^{a+b} 4 \Gamma(2 a+2) \Gamma(2 b+2) x y}{\Gamma(a+1) \Gamma(b+1)}\right) .
\end{aligned}
$$

(ii) If $a, b, c, d<0$ and $0<x^{2}, y^{2}, z^{2}, w^{2}<1$, then

$$
\begin{aligned}
( & \left.\frac{1}{1-\frac{2}{3} a x^{2}-\frac{2}{3} b y^{2}}\right)\left(\frac{(-1)^{a+b} \Gamma(c+1) \Gamma(d+1) \Gamma(2 a+2) \Gamma(2 b+2) x y}{3}\right) \\
& <\frac{H_{2 a+1,2 b+1}(x, y)}{H_{2 c+1,2 d+1}(z, w)} \\
& <\left(\frac{1-\frac{4}{3} a x^{2}-\frac{4}{3} b y^{2}}{1-\frac{2}{3} c z^{2}-\frac{2}{3} d w^{2}}\right)\left(\frac{(-1)^{a+b} \Gamma(c+1) \Gamma(d+1) \Gamma(2 a+2) \Gamma(2 b+2) x y}{(-1)^{c+d} \Gamma(a+1) \Gamma(b+1) \Gamma(2 c+2) \Gamma(2 d+2) z w}\right) .
\end{aligned}
$$

(iii) If $-\frac{3}{2}<a, b, c, d<0, x^{2}, y^{2}>0$ and $0<z^{2}, w^{2}<1$, then

$$
\begin{aligned}
& \left(\frac{1+\frac{2}{3} a x^{2}+\frac{2}{3} b y^{2}}{1+\frac{2}{3} c z^{2}+\frac{2}{3} d w^{2}+\frac{2 c(c-1)}{15} z^{4}+\frac{4 c d}{9} z^{2} w^{2}+\frac{2 d(d-1)}{15} w^{4}}\right) \\
& \quad \times\left(\frac{(-1)^{a+b} \Gamma(c+1) \Gamma(d+1) \Gamma(2 a+2) \Gamma(2 b+2) x y}{(-1)^{c+d} \Gamma(a+1) \Gamma(b+1) \Gamma(2 c+2) \Gamma(2 d+2) z w}\right) \\
& <\frac{H_{2 a+1,2 b+1}(-x,-y)}{H_{2 c+1,2 d+1}(-z,-w)}<\left(\frac{1+\frac{2}{3} a x^{2}+\frac{2}{3} b y^{2}+\frac{2 a(a-1)}{15} x^{4}+\frac{4 a b}{9} x^{2} y^{2}+\frac{2 b(b-1)}{15} y^{4}}{1+\frac{2}{3} c z^{2}+\frac{2}{3} d w^{2}}\right) \\
& \quad \times\left(\frac{(-1)^{a+b} \Gamma(c+1) \Gamma(d+1) \Gamma(2 a+2) \Gamma(2 b+2) x y}{(-1)^{c+d} \Gamma(a+1) \Gamma(b+1) \Gamma(2 c+2) \Gamma(2 d+2) z w}\right) .
\end{aligned}
$$


Proof Similarly the results (6.17)-(6.19) can also be obtained with the help of (1.7)-(1.10) and (6.16).

In conclusion, Luke's technique is to a large extent successful in obtaining inequalities for the confluent hypergeometric function. The theorems developed herein seem sufficient to indicate the general nature of expected results and we do not further pursue the subject. Here, we have obtained inequalities for simple Laguerre, Laguerre, pseudo-Laguerre, Shively's pseudo-Laguerre, and Hermite functions from a well-known result for confluent hypergeometric functions and their confluent cases. In a future work, we intend to investigate this aspect of the subject and to apply our techniques to develop inequalities for hypergeometric functions of several variables.

\section{Competing interests}

The author declares that they have no competing interests.

\section{Acknowledgements}

The author expresses sincere appreciation to Dr. Mohamed Saleh Metwally (Department of Mathematics, Faculty of Science (Suez), Suez Canal University, Egypt), and Dr. Mahmoud Tawfik Mohamed (Department of Mathematics, Faculty of Science (New Valley), Assiut University, New Valley, EL-Kharga 72111, Egypt) for their kind interests, encouragement, help, suggestions, comments and the investigations for this series of papers. The author would like to thank the anonymous referees for valuable comments and suggestions, which have led to a better presentation of the paper.

Received: 1 October 2014 Accepted: 1 May 2015 Published online: 16 May 2015

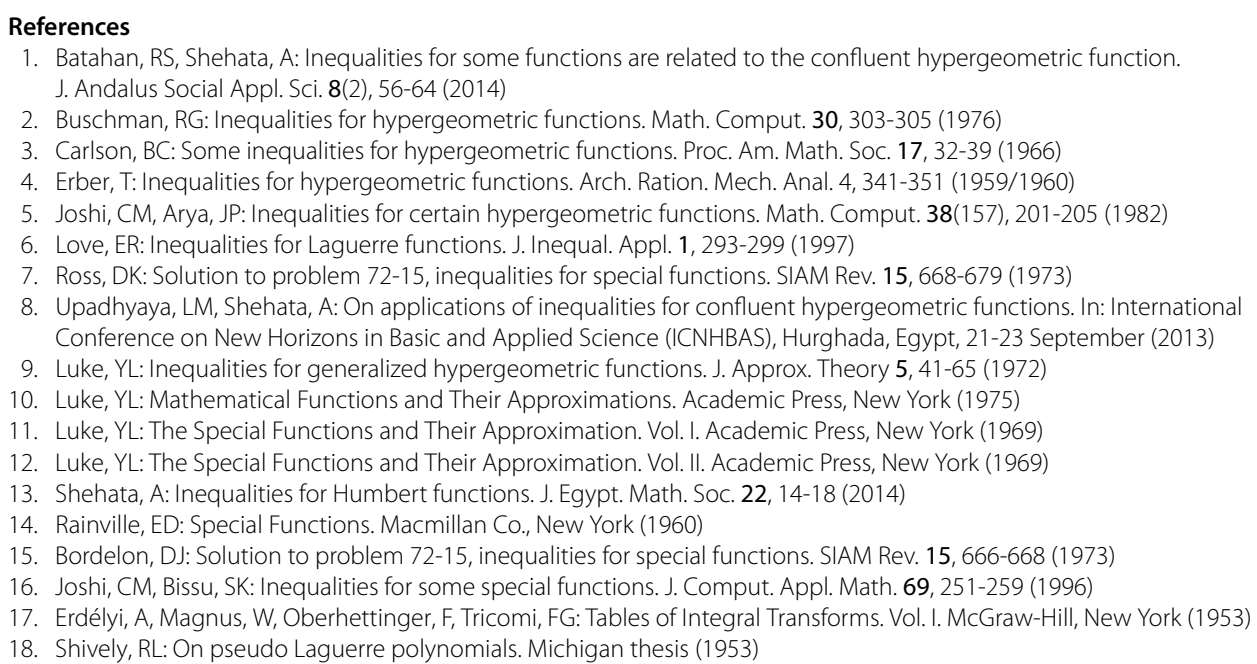

\section{Submit your manuscript to a SpringerOpen ${ }^{\circ}$ journal and benefit from:}

- Convenient online submission

- Rigorous peer review

- Immediate publication on acceptance

Open access: articles freely available online

- High visibility within the field

- Retaining the copyright to your article 